\title{
Crosswise Model to Assess Sensitive Issues: A Study on Prevalence of Drug Abuse Among University Students of Iran
}

\author{
Ahmad Khosravi ${ }^{1, *}$; Seyed Abbas Mousavi ${ }^{2}$; Reza Chaman ${ }^{3}$; Faride Khosravi ${ }^{4}$; Mohammad \\ Amiri $^{5}$; Mansour Shamsipour ${ }^{6}$ \\ ${ }^{1}$ Center for Health Related Social and Behavioral Sciences Research, Shahroud University of Medical Sciences, Shahroud, IR Iran \\ ${ }_{2}^{2}$ Golestan Research Center of Psychiatry, Golestan University of Medical Sciences, Gorgan, IR Iran \\ ${ }_{4}^{3}$ Department of Social Medicine, Yasuj University of Medical Sciences, Yasuj, IR Iran \\ 4 Department of Social Medicine, Yasuj University of Medical Sciences, Yasuj, IR Iran
${ }_{5}$ Department of Biostatics, Mazandaran University of Medical Sciences, Sari, IR Iran \\ ${ }_{5}^{5}$ School of Public Health, Shahroud University of Medical Sciences, Shahroud, IR Iran \\ 6 Department of Epidemiology and Biostatistics, School of Public Health, Tehran University of Medical Sciences, Tehran, IR Iran
${ }^{2}$, \\ *Corresponding author: Ahmad Khosravi, Center for Health Related Social and Behavioral Sciences Research, Shahroud University of Medical Sciences, Shahroud, IR Iran. Tel: \\ +98-2733394499, Fax:+98-2733394800, E-mail: khosravi2000us@yahoo.com
}

Received: October 7, 2014; Revised: December 18, 2014; Accepted: December 24, 2014

\begin{abstract}
Background: Precise assessment of the prevalence of illicit drug use, face various methodological challenges.
Objectives: The current study aimed to investigate the prevalence of illicit drug use among students studying at Universities in Shahroud (Northeast of Iran) through crosswise method.

Patients and Methods: Participants of this cross-sectional study were 1646 students at Universities in Shahroud. The data collection instrument was a questionnaire designed based on crosswise model (CM).

Results: The results of the study showed that $19 \%$ of students used at least one kind of illicit drug and $14.9 \%$ used opium residue once in their life. Moreover, $3.5 \%$ of students used drug and 3\% used opium residue during the last month; $40.3 \%$ of the students admitted that they fully understood the instruction of the questionnaire; $9.6 \%$ said that did not comprehend the instruction at all, and 38.1\% believed they partly understood the instruction. The result showed that $33.7 \%$ of the students fully trusted, $39.8 \%$ partly trusted, and $18.4 \%$ poorly trusted this method. There was a significant relationship between comprehension level and trust in $\mathrm{CM}(\mathrm{P}<0.001)$.

Conclusions: This method can be appropriate to estimate sensitive issues; however, lack of understanding the method or doubting the confidentiality of the responses can lead to bias in the results. That is to say, if the instructions are introduced better, the level of trusting the method will increase and accordingly more reliable responses can be obtained. More studies under controlled conditions are required to interpret the findings of the current study better.
\end{abstract}

Keywords: Drug Abuse; Illicit Drug; Iran

\section{Background}

One of the important information sources to help the authorities decide on the public health issues and their relation with other social issues is eliciting data from the target individuals and collecting their responses. Yet, direct questions on the innermost private characteristics of people, their unacceptable social behaviors, illicit behaviors, etc., which are technically called sensitive issues, face various methodological challenges. Since different reasons such as disturbance, disclosure, susceptibility, and social acceptance people may avoid taking part in the study; or some people may participate in the study but skip sensitive items or provide inaccurate responses. Such errors may cause biases like social desirability bias and consequently desirable social behaviors such as using seatbelt, voting and exercise can be over reported and undesirable behaviors such as drug abuse, abortion, and high risk sex behavior can be prone to under reporting (1). There are different strategies, which aim to decrease such measurement errors and biases and increase the reliability of people responses. Randomized Response Technique (RRT) designed by Varner in 1965 can be viewed as the earliest attempt to obtain valid information to estimate the proportion of a sensitive behavior in a population without revealing the true status of the respondents. Different versions of Randomized Response Technique are evolved and introduced through time (2-4). In all these procedures, in addition to the item on the sensitive behavior, an insensitive item which is independent of the sensitive item is given to the respondent. Based on a randomized instrument, the distribution of which is known to the researcher, the respondent decides which item to answer. Therefore, the meaning of the responses depends on the results of the random instrument, which is unknown to the researcher and responding does not indicate anything about the true status of the sensitive behavior.

Being aware of this anonymity, the respondent is ex-

Copyright (C) 2015, Zahedan University of Medical Sciences. This is an open-access article distributed under the terms of the Creative Commons Attribution-NonCommercial 4.0 International License (http://creativecommons.org/licenses/by-nc/4.0/) which permits copy and redistribute the material just in noncommercial usages, provided the original work is properly cited. 
pected to provide more honest responses to direct questions. Yet, all randomized response techniques depend on the randomized instrument used by the interviewer, understanding and performing some of the procedures are difficult and costly and not recursive or repeatable. To overcome some of the deficiencies in RRT, for a two status sensitive item, two non-randomized response techniques called crosswise (CW) and triangular procedures are recently developed by Yu et al. (5), which should be appropriate to solve some of the problems of randomized response models. As these authors maintain, these two models are appropriate both for face to face interviews and for selfadministered interviews. As these two models do not need any randomized instruments, their administration is easier than RRT models. Another advantage of these models is that none of the respondents directly answers the sensitive items. However, in RRT models, at least some of the respondents should directly answer the sensitive items (5). Using prior knowledge of the population prevalence for an insensitive question led to the development of a number of competing techniques. In these models, the non-sensitive question is outside the researcher's control, independent of the research question but the population prevalence such as birth month or season, geographical location for the person or a family member is already established. Crosswise model uses a combination of sensitive and an insensitive questions with known population prevalence (5). Although Yu et al. (5), introduced the theoretical components and analysis of these models, the practical application of these two methods are not yet known, and to the best knowledge of the present study authors, these two methods are not tested in the domain of drug abuse. Drug abuse by university students worldwide, and alcohol use in Iran are regarded as sensitive issues and many researchers investigated the prevalence of drug abuse among students. However, as many of these researchers admit, due to the sensitivity of the issue and mistrust of the respondents, the prevalence of drug abuse is much higher than reported (6-13). Due to the anonymity which CW model entails for the participants, in the current study it was assumed that anonymity leads to an increase in the trust of the respondents and CW model can provide estimates different from those of the direct methods.

In this method, respondents receive two items with yes-no options. One item is sensitive and the other two option item is insensitive and independent of the sensitive item. In this model, it is assumed that the probability of positive response to insensitive item is already known to the researcher. Of course the probability distribution should not be the same for the insensitive item. To respond the items, the respondent is asked to choose " $\mathrm{A}$ " if the answers to both items are the same (both items yes or both no), and to choose "B" if the answers to both items are not the same (one answer is yes and the other is no). Assuming that the respondent honestly answers the items, an unbiased estimation of the prevalence of the sensitive trait is obtained as follows (Equation 1) (5): $\lambda=$ the proportion of those who choose $\mathrm{A}$

$\pi=$ the proportion of those who have sensitive behavior.

$\mathrm{p}=$ the probability of positive response to insensitive item $p \neq 0.5$.

$$
\lambda=\mathrm{p} \pi+(1-p)(1-\pi)
$$

An orthogonal estimate is obtained through Equation 2:

$$
\tau=\frac{\lambda+p-1}{2 p-1},(p \neq 0.5)
$$

The variance of $\pi$ is calculated as follows (Equation 3). It should be noted that the second part of the equation is due to the insensitive item.

$$
\operatorname{Var}(\pi)=\left[\frac{\pi(1-\pi)}{n}\right]+\left[\frac{p(1-p)}{n(2 p-1)^{2}}\right]
$$

\section{Objectives}

The current study aimed to investigate the prevalence of drug abuse among students at Universities in Shahroud through $\mathrm{CM}$ and to evaluate the comprehension and trust levels of the participants in this method. The results can be helpful to improve the research methods in addiction studies, evaluate anti-drug programs, and plan for better tackling of drug related problems.

\section{Patients and Methods}

The participants in this cross-sectional study were 1644 students of Shahroud University of Medical Sciences, Shahroud University of Technology, Shahroud Islamic Azad and Payame Noor Universities. Fifty classes were selected from these Universities through stratified and cluster random sampling. Approximately $10 \%$ of the students attending Shahroud universities were selected. All the students attending classroom sessions during December 2011 to January 2012 were asked to fill out a selfadministered questionnaire. Questionnaires were distributed at the beginning of each class after explanation about the CW model, and the students were given time to fill out the questionnaires.

\subsection{Estimation of the sample size}

The current study used the sample size formula introduced by the developers of the model (Equation 4):

$$
\mathrm{n}=\frac{\mathrm{Z}_{\frac{\mathrm{a}^{2}}{2}}}{\mathrm{~L}_{0}(2 \mathrm{p}-1)}
$$


$\mathrm{L}_{0}=0.106$ is the maximum confidence interval around the prevalence of the sensitive trait $(\pi)$, which depends on the precision level intended by the researcher.

$\mathrm{P}=0.26$ is the probability of positive response to the insensitive items, which is already known to the researchers. The sample size was estimated to be 1650 , according to the mentioned parameters. The Ethics Committee of Shahroud University of Medical Sciences approved the study protocol (No. 900.20). Oral informed consent was obtained after explaining the objectives of the study, and the people agreed to participate.

\subsection{Instrument}

The study instrument was designed based on crosswise model as follows. At the beginning of the questionnaire, instructions were provided on how to answer the items. Then there were the demographic items. It was tried to select items in a way which would produce the minimal sensitivity in the participants. These items were limited to asking about gender, place of residence, and marital status. The rest of the questionnaire included six pairs of items. The main results were last month and lifetime using one of the narcotic drugs and last month and lifetime using of opium or burned opium. Each sensitive item was accompanied by an insensitive item with a known probability distribution (Table 1). Assuming equal distribution of identical distribution of birth in seasons throughout a year, one of the items was on the date of birth. Moreover, according to a study which investigated the distribution of major blood types in different geographical areas and among different racial-ethic groups of Iran, $24.36 \%$ of people have blood group $\mathrm{B}(14)$. Therefore one insensitive item was "Is your blood group B?" Moreover, assuming that the distribution of numbers (including $0,1,2,3,4,5$, $6,7,8,9$ ) in ATM passwords, building numbers, telephone numbers and childbirth through year are equal, insensitive items that were shown in the Table 1 were developed. In order to test the quality appraisal of the obtained responses, to examine the internal consistency and to experimentally compare the estimates of the model with the expected values, in one pair (No.2 in Table 1); both items were selected as insensitive with the expected probability distribution. Moreover, since lack of comprehension of the instruction to complete the questionnaire and the degree of trust were among major bias sources, the two items on these issues were put at the end of the questionnaire.

\subsection{Data Analysis}

The data were analyzed through the equations already introduced in this paper. To investigate the association between comprehending the instruction for crosswise model and the trust of respondents in it, Chi-square test was used. All calculations were performed through Excel and R-package.

\section{Results}

Out of 1644 participants, $49.5 \%(n=814)$ were male and $50.4 \%(n=830)$ female.

\subsection{Estimates Obtained Through Crosswise Model}

Table 2 displays the prevalence and confidence interval for using each of the interrogated cases.

Table 1. Items in the Crosswise Model Questionnaire

\begin{tabular}{ll}
\hline Item & Question \\
\hline
\end{tabular}

1 Think of the password of one of your ATM cards, which you use more often.

Is the final figure of this password one of the numbers 5,6 , or 7 ?

Have you over the last month used one of the narcotic drugs (cannabis, opium, opium extract, crack, heroin, glass, ecstasy)?

Is the final figure of your ID number 2,4 , or 6 ?

Were you born in summer?

3 Think of the number of your father's or an acquaintance's house and do not change it.

Is the rightmost figure 2,4 , or 6 ?

Have you used opium during the last month?

4 Think of a friend or acquaintance whom you know very well and know when exactly he was born.

Is his or her birthday between the first and the tenth day of the month?

Have you ever used any narcotic drug (cannabis, opium, opium extract, crack, heroin, glass, ecstasy)?

5 Think of one of your friends that you know his/her mobile number.

Is the rightmost figure of his/her mobile number 2,4 or 6 ?

Have you ever used opium or Burned opium? 
Khosravi A et al.

\begin{tabular}{|c|c|c|c|}
\hline Items & Male $(\mathrm{N}=\mathbf{8 1 4})$ & Female $(N=830)$ & Total $(N=1644)$ \\
\hline Use of illicit drugs throughout the lifetime & $21.4(13.1-29.8)$ & $16.9(8.7-25.1)$ & $19.0(13.1-24.8)$ \\
\hline Use of illicit drugs during the last month & $6.1(0-14.1)$ & $0.9(0-8.7)$ & $3.5(0-9.1)$ \\
\hline Use of opium or its burnt during the last month & $3.9(0-11.9)$ & $2.1(0-10.0)$ & $3.0(0-8.6)$ \\
\hline Use of opium or its burnt throughout the lifetime & $18.9(10.7-27.3)$ & $11.4(3.4-19.5)$ & $14.9(9.1-20.7)$ \\
\hline
\end{tabular}

Data are presented as \% $(95 \% \mathrm{CI})$.

Table 3. Experimental Estimation With Crosswise Model ${ }^{\text {a }}$

\begin{tabular}{lcccc}
\hline & Total & Male & Female & Expected Distribution \\
\hline Item1: Was the person born in summer? & $27(22-33)$ & $25(17-33)$ & $30(21-38)$ & $25 \%$ \\
\hline
\end{tabular}

${ }^{a}$ Data are presented as \% (95\%CI).

The results of the study showed that $19 \%$ of the students used at least one kind of illicit drug and 14.9\% used opium residue (yen-shee) once over their life. There was no statistically significant difference between males and females in this regard. Moreover, $3.5 \%$ of the students used drugs and 3\% used opium residue during the last month. There was no a statistically significant difference between males and females in this regard $(\mathrm{P}<0.05)$.

Regarding the instruction comprehension, $40.27 \%$ of the students reported that they fully understood the questionnaire instructions, 38.09\% reported partial understanding, and $12.02 \%$ reported little understanding, and $9.59 \%$ reported no understanding of the instructions.

With reference to the trust in the CM method, $33.71 \%$ of the students said they highly trusted the model, while 39.85\% moderately trusted, and $8.01 \%$ mistrusted the method. Moreover, there was a statistically significant relationship between understanding the method and trusting it; hence those who understood the method better had more trust in it.

The results of assessing of internal consistency of the questionnaires are displayed in Table 3. If the distribution estimate is the birth season of individuals (in case of asking about 2, 4, and 6 as the final figure of ID card is regarded as insensitive question), the model estimated it for the total population to be $27 \%$ (95\% for CI: 22 - 33), for females 30\% (95\%for CI: 21 - 38) and for males 25\% (95\% for CI: 17 - 33).

\section{Discussion}

The current study aimed to evaluate the application of crosswise model in estimating the proportion of drug abuse. In general, it can be said that the results of the current study on narcotic drugs and burnt opium abuse over life and during the last month are consistent with the findings of other studies carried out among students of Iranian Universities with direct questionnaires (7, 8, $10,15)$. In the previous studies on drug abuse, the prevalence of drug abuse over life and during the last month was greater among males and showed a significant difference with that of females. However, although instant es- timation of these prevalence figures in CWM was greater in males, the differences were not significant. Moreover, opium or burnt opium abuse over life was reported more among males, but there was no statistically significant difference with that of females.

Since CWM requires a large sample size to provide consistent estimates, and with regard to the high standard error in the two groups (especially males), the obtained result can be a matter of chance and due to small sample size in the two levels. On the other hand, since drug abuse by females is denounced more in the society than its abuse by males, it is possible that females trusted more in CWM and provided more accurate responses as compared to what other studies reported, and in fact it can be one of the advantages of CWM. Moreover, in the recent years there has been an increase in the students' tendency, especially females, towards narcotic drugs, smoking, and alcoholic drinks, which has led to concerns in student environments; it can also be one of the reasons for apparently incongruent results.

Another result of the current study was the low confidence interval for the prevalence of drug abuse during the last month (0.95 CI: 0-9.1). This can be by chancing or the respondents may not have correctly understood the instruction. In the insensitive item, which accompanied the item on drug abuse during the last month, it was assumed that the final figure in ATM passwords is equally distributed. However, since people usually change their passwords, there may have been a preference for certain numbers for which the distribution was not equal and accordingly introduced error into the estimations. Estimation of the lifetime opium or burnt opium abuse was based on the insensitive item for which the equal distribution of the final figure in the mobile phone number was assumed.

One of the indicators of the evaluation model was the level of comprehending the instructions as well as the level of the participants trust in crosswise model and the association between them; $40.27 \%$ of the students said that they had fully understood the instructions. The reason for lack of understanding the instructions in $9.59 \%$ 
or partial understanding in $38.85 \%$ of the students can be the fatigue after some classes.

Another important aspect of evaluating the success of this method was the number of participants who trusted that their personal information would be kept confidential. The results showed that $33.71 \%$ of the students highly trusted, 39.85\% moderately trusted, $18.4 \%$ poorly trusted, and $8.01 \%$ distrusted this method. Since a significant relationship was found between understanding the method and trusting it, it can be said that improving the understanding of the instructions can result in an increase in the level of trust in the method and more accurate responses. It further indicates that the instructions provided by the researchers were not appropriate.

In this method of responding, the respondent cannot take a self-protection strategy and give a negative answer to a question. As a result, it reduces the self-protection bias. This is in fact one of the advantages of CW model over RRT techniques. Since in this method the estimations are based on peripheral techniques, and the normality of the data requires a large sample size, the present results indicated that for rare attributes with a skewed likelihood distribution, the method cannot provide consistent estimates. In these cases, Bayesian methods can be used. These findings were consistent with the findings of Ben John et al. on plagiarism (16). One limitation in evaluating the model was the inaccessibility of findings on trust in a direct questionnaire, which can be compared with CWM. In this study six pairs of items were designed. It is possible that some students thought that some items were repetitious and the researchers intended to ultimately find an association between these items and detect their response and it influenced their trust. Therefore other studies with fewer pairs of items are suggested. Other studies with direct questionnaires and other NRR designs such as triangular and Hidden Sensitivity (HS) models can be carried out and their results can be compared to one another. Other populations such as school students can also be studied using these designs. In this way, the results can be judged soundly.

One of the most important and complicated parts of this model was selecting an insensitive item with a clear probability distribution appropriate for the population under study. In addition to meeting the conditions of the model, such items should be designed in a way which makes it impossible for the researcher to find their answers and only the respondent himself should be aware of the answer. Since the insensitive items used in the study may not have corresponded to the assumptions, the results of the study may be distorted by this incongruence. Moreover, high rate of lack of comprehension and distrust in the model showed that this model was not understandable to the students. This can also result from the complexity of the model or inaccuracy of the researchers to follow the instructions. Another issue was that this model proves inconsistent estimates for traits with almost zero prevalence, such as the prevalence of drug abuse in females. In sum, more studies with other populations are required to better compare this model with the other models to detect its possible superiorities. The main limitation was lack of understanding the method or doubting the confidentiality of the responses that can lead to bias in the results. The current study findings suggest that the CWM is a fruitful data collection method for sensitive topics such as drug abuse. Also the obtained results showed a good estimation for this method when two insensitive questions were used. Further studies comparing the direct and indirect methods to estimate the prevalence of drug abuse in students are desirable.

\section{Acknowledgements}

We also wish to thank all of the students for their valuable collaboration with this study.

\section{Authors' Contributions}

Reza Chaman, Mansour Shamsipour, Seyed Abbas Mousavi and Ahmad Khosravi contributed to the study concept, design, data gathering, data analysis, interpretation of data and drafting the manuscript. Mohammad Amiri and Faride Khosravi contributed to the data gathering, and reviewing the manuscript.

\section{Funding/Supports}

This study was supported by the Research council at Shahroud University of Medical Sciences, grant No 9005.

\section{References}

1. Tourangeau R, Yan T. Sensitive questions in surveys. Psychol Bull. 2007;133(5):859-83.

2. Cruyff MJLF, van den Hout A, van der Heijden PGM, Bockenholt U. Log-linear randomized-response models taking self-protective response behavior into account. Socio Meth Res. 2007; 36(2):266-82.

3. Maddala GS. Limited-Dependent and Qualitative Variables in Econometrics. Cambridge University Press; 1986.

4. Weissman AN, Steer RA, Lipton DS. Estimating illicit drug use through telephone interviews and the randomized response technique. Drug Alcohol Depend. 1986;18(3):225-33.

5. Yu JW, Tian GL, Tang ML. Two new models for survey sampling with sensitive characteristic: design and analysis. Metrika. 2008;67(3):251-63.

6. Agahi C, Spencer C. Patterns of drug use among secondary school children in post-revolutionary Iran. Drug Alcohol Depend. 1982;9(3):235-42

7. Ahmadi J, Benrazavi L, Ghanizadeh A. Substance abuse among contemporary Iranian medical students and medical patients. $J$ Nerv Ment Dis. 2001;189(12):860-1.

8. Ahmadi J, Javadpour A. Assessing substance use among Iranian health care students. Eur J Psychia. 2002;16(3):174-7.

9. Ahmadi J, Maharlooy N, Alishahi M. Substance abuse: prevalence in a sample of nursing students. IClin Nurs. 2004;13(1):60-4.

10. Ahmadi J, Fallahzadeh H, Salimi A, Rahimian M, Salehi V, Khaghani M, et al. Analysis of opium use by students of medical sciences. JClin Nurs. 2006;15(4):379-86.

11. Mohammad Poorasl A, Vahidi R, Fakhari A, Rostami F, Dastghiri S. Substance abuse in Iranian high school students. Addict Behav. 2007;32(3):622-7.

12. Najafi K, Zarrabi H, Shirazi M, Fekri F, Mohseni R. Prevalence of 


\section{Khosravi A et al.}

substance use among Iranian high school students in 20052006. Kuwait Med J. 2009;41(1):20-5.

13. Mohammadpoorasl A, Fakhari A, Rostami F, Vahidi R. Predicting the initiation of substance abuse in Iranian adolescents. Addict Behav. 2007;32(12):3153-9.

14. Pourfathollah AA, Oody A, Honarkaran N. Geographical distribution of $\mathrm{ABO}$ and $\mathrm{Rh}(\mathrm{D})$ blood groups among Iranian blood do- nors in the year 1361 (1982) as compared with that of the year 1380 (2001). Sci J Blood Transfus Organ. 2004;1(1):11-7.

15. Ghanizadeh A. Shiraz University students' attitude towards drugs: an exploratory study. East Mediterr Health J. 2001;7(3):452-60.

16. Jann B, Jerke J, Krumpal I. Asking sensitive questions using the crosswise model an experimental survey measuring plagiarism. Public Opin Q. 2011;76(1):32-49. 